\title{
Implementation intention encoding does not automatize prospective memory responding
}

\author{
Mark A. McDaniel ANd Michael K. Scullin \\ Washington University, St. Louis, Missouri
}

\begin{abstract}
An implementation intention encoding, one that specifies the concrete situation that is appropriate for initiating an intended action and links that situational cue to the intended action, has been shown to improve prospective memory. One proposed mechanism is that implementation intentions create automatized prospective remembering. This view anticipates that implementation intentions should prevent prospective memory decline in highly cognitively demanding situations. Contrary to this expectation, although implementation intention encoding enhanced prospective memory (Experiments 1 and 2), implementation-intention encoding did not buffer against significant prospective memory decline in high-cognitive-demand conditions (Experiments 1-3), and in Experiment 3, implementation intention encoding produced lower levels of prospective memory performance than did behavioral practice in the high-cognitive-demand situation. We suggest that although implementation intentions may stimulate a strong associative encoding (between an anticipated environmental cue and an intended action), that encoding does not support a completely automatized prospective memory response.
\end{abstract}

Remembering to execute previously formed intentions at an appropriate moment is a ubiquitous aspect of human functioning (for purposes of exposition, we label this prospective memory). From managing household activities (remembering to pay bills) to maintaining social relations (remembering to send a friend a card on her birthday) to regulating health-related needs (remembering to take medication), good prospective memory is critical to normal functioning. The consequences of prospective memory failures can range from social discomfort and embarrassment (such as forgetting to pick up one's child; Winograd, 1988; see also McDaniel \& Einstein, 2007) to threats to health (such as forgetting to remove surgical instruments from a patient or forgetting to drop one's child off at day care; see McDaniel \& Einstein, 2007, chap. 9). Accordingly, understanding prospective memory and proposed techniques for improving prospective remembering is an important challenge for memory researchers.

One of the most promising approaches identified in the literature for improving prospective memory is an encoding technique that involves (1) specifying the concrete situation that is appropriate for initiating the intended action and (2) linking the specific situational cue to the intended action (Gollwitzer, 1999). This technique prescribes an encoding of the form "if I encounter X then I will do Y," an encoding that Gollwitzer termed an implementation intention. Implementation intentions have been shown to significantly increase the likelihood that intended actions will be performed across an impressive range of naturalistic tasks (Liu \& Park, 2004; Milne, Orbell, \& Sheeran, 2002; Orbell, Hodgkins, \& Sheeran, 1997; Sheeran \& Or- bell, 1999). As just one example, one study reported that $91 \%$ of the college student participants who formed an implementation intention engaged in vigorous exercise for 20 min during the upcoming week, whereas only $29 \%$ of the control participants (who simply formed the intention to exercise in the upcoming week) adhered to their intention. Implementation intentions are also effective for improving performance on laboratory prospective memory tasks (Cohen \& Gollwitzer, 2008; McDaniel, Howard, \& Butler, 2008).

The most elaborated and prominent theoretical account for the prospective memory improvements produced by implementation intentions has been offered by Gollwitzer (1999). On this account, a number of mechanisms converge to create automatized initiation of the intention upon encountering the specified situation. First, the mental representation of the anticipated situational cue is thought to be highly activated so that it is easily accessible. Second, once the situational cue is presented (e.g., in a laboratory experiment), it stimulates execution of the intended action immediately, efficiently (other things can be done at the same time), and without conscious intent (see Gollwitzer, 1999, p. 498; see also Bayer, Achtziger, Gollwitzer, \& Moskowitz, 2009). Of interest in the present study is the following key implication: The positive effects of implementation intentions should prevail even when cognitive resources are limited or challenged.

A related, but less strong, formulation is that an implementation intention is effective because it fosters a robust associative encoding between the critical situation and the intention (see, e.g., Cohen \& Gollwitzer, 2008; Mc-

M. A. McDaniel, mmcdanie@artsci.wustl.edu 
Daniel, Howard, \& Butler, 2008). Theoretically, a robust associative encoding may stimulate spontaneous retrieval of the intended action (McDaniel, Guynn, Einstein, \& Breneiser, 2004), but spontaneous retrieval itself need not produce efficient, immediate initiation of the action that occurs without conscious intent. On this formulation, other resource-demanding processes are still needed (even with implementation intention encoding) to manage and coordinate the execution of the intended action in the face of competing demands from ongoing activities (see Einstein, Smith, McDaniel, \& Shaw, 1997; Marsh, Hicks, \& Watson, 2002; McDaniel, Robinson-Riegler, \& Einstein, 1998). According to the associative encoding view, if the demands of the ongoing activity are not overly challenging (e.g., a typical ongoing activity), resources should be available to interpret the retrieved intention and coordinate its execution with ongoing demands (cf. Marsh et al., 2002). Under such conditions, the presumed associative retrieval advantage of an implementation intention will improve prospective memory. The critical departure from the automatization view outlined above is that implementation intentions would not necessarily be expected to buffer against prospective memory failures under sufficiently (cognitively) demanding contexts (we expand on the theoretical explanation for this expectation in the following Discussion sections).

Although minimal, the published evidence is consistent with the implication that implementation intentions will benefit prospective memory even when cognitive resources are challenged (e.g., by increasing the cognitive demands of the ongoing activity in which the prospective memory task is embedded). In Brandstätter, Lengfelder, and Gollwitzer (2001), numbers and single digits appeared on a screen, and participants had to press a key when digits appeared. In addition, some participants formed an implementation intention to press the target key particularly quickly (the prospective memory task) when they saw a specified number (e.g., "3"). Other participants (the control group) were familiarized with the specified number and also were instructed to prepare themselves for the presentation of this number (these participants also had to press the target key). The important design feature was that this task was presented in the context of another task (the primary task, which varied across studies from a tracking task to working on meaningless syllables), with the primary task manipulated to be either easy or difficult. The assumption was that the difficult version of the primary task would result in a high-cognitive-demand situation. The general finding was that even under high cognitive demand, implementation intentions produced faster responding to the specified number than did familiarization. These results have been interpreted as indicating that the use of implementation intentions results in effortless, automatic processing (Gollwitzer, 1999).

A more recent laboratory study examined the effects of implementation intentions on the more typical prospective memory measure of successfully remembering to perform an intended action. In some conditions, the high cognitive demand of generating random digit sequences was added to the ongoing activity (rating the character- istics of words' referents). Critically, when implementation intentions were formed, prospective memory performance showed no decline in the high-cognitive-demand conditions, relative to the standard-cognitive-demand condition (without random-digit generation); by contrast when standard prospective memory instructions were given, prospective memory significantly declined in the high cognitive demand condition (McDaniel, Howard, \& Butler, 2008, Experiment 2). This pattern is consistent with the idea that implementation intention encoding produces automatized initiation of an intended action upon presentation of the situation cue specified in the implementation intention.

Although existing findings are suggestive, more research is warranted before it can be safely concluded that implementation intention encoding supports superior (and intact) prospective remembering even under high cognitive demand. The studies just noted were limited in several ways. In McDaniel, Howard, and Butler (2008), the experimenter-controlled presentation rate of the ongoing activity may have been slow enough that the full challenge of the high-cognitive-demand conditions was somewhat negated. This possibility is supported by relatively high prospective memory performance in the standard prospective memory conditions under high cognitive demand (random-digit generation). In Brandstätter et al. (2001, Study 3 ), the success of the cognitive demand manipulation was assessed through participants' self-reports, and the rated difference across cognitive demand conditions was not significant, thereby raising issues about whether cognitive demand was sufficiently varied. In Study 4, the cognitive demand manipulation was objectively confirmed; however, the absence of a significant load effect on the prospective memory task (speed of responding to the specified number) in the implementation intention condition may have been paralleled by the absence of a load effect in the familiarization control. The pertinent analyses were not reported, but even in the familiarization control, the means do not reveal a robust slowdown under load (relative to noncritical numbers; see Brandstätter et al., 2001, Figure 2, comparing critical with noncritical numbers). Thus, either the load manipulation was again not potent enough (see also McDaniel, Howard, \& Butler, 2008, Experiment 1), or the particular task of interest (fast responding to a specified number) was a behavior that did not demand significant cognitive resources.

In the following experiments, we improved the previous paradigms by increasing the challenge of the experimental tasks, thereby allowing stronger tests of the notion that implementation intention encoding produces automatic action initiation and, consequently, more immunity to high cognitive demands than do standard prospective memory encodings.

\section{EXPERIMENT 1}

A key feature of typical prospective memory paradigms is that the prospective memory task is embedded in an ongoing activity designed to keep participants busily engaged. In the present experiment, we selected an ongo- 
ing activity that required a normatively correct answer, rather than a response based on subjective impressions (as was used in McDaniel, Howard, \& Butler, 2008). On each trial, participants were given a particular category label paired with another word, and their task was to respond whether the given word was a member of the indicated category. We reasoned that this judgment could not be as easily shortcut under high cognitive demand as would a subjective rating (used in McDaniel, Howard, \& Butler, 2008). To further discourage displacement of resources from the ongoing activity during high cognitive demand (which could, in turn, be deployed for prospective memory), we made the ongoing activity subject paced and emphasized to the participants that it was important for them to respond as quickly as possible on each trial (rather than allowing the participants a relatively comfortable 5-sec per item, as in McDaniel, Howard, \& Butler, 2008). In the high-cognitive-demand condition, the participants were required to perform the random-number generation task in addition to the ongoing category decision task.

On the basis of existing findings from experiments in which random number generation was used to create high cognitive demand (Marsh \& Hicks, 1998; McDaniel, Howard, \& Butler, 2008), we expected that with standard prospective memory encoding instructions, prospective memory performance would significantly decline under high-cognitive-demand conditions, relative to standardcognitive-demand (without random-number generation) conditions. More important, on the view that implementation intentions support effortless, automatic execution of intended actions, prospective memory performance for a group using an implementation intention should not significantly decline in the high-cognitive-demand condition (relative to the standard-cognitive-demand condition).

Alternatively, as was outlined earlier, implementation intentions may primarily enhance the associative encoding between a prospective memory cue and the intended action (Cohen \& Gollwitzer, 2008; McDaniel, Howard, \& Butler, 2008). The increased associative encoding could support superior prospective memory (relative to a typical prospective memory instruction) under relatively standard cognitive demands, but under high cognitive demands, the implementation intention group should not be significantly advantaged, relative to the typical-instruction group (because, as will be discussed below, increased associative encoding of a cue and action is not sufficient to support automatic execution of the prospective memory).

Before presenting this first experiment, several comments regarding the prospective memory instructions deserve mention. The first concerns the particular form of the implementation intention instructions. The fundamental format specified by Gollwitzer and colleagues is the participant's verbal specification that "when [if] a specific event cue appears, [then] I will perform a particular behavior." However, experimental instantiations of implementation-intention encoding instructions have varied widely (see, e.g., Brandstätter et al., 2001; Chasteen, Park, \& Schwarz, 2001; Gollwitzer \& Brandstätter, 1997; Milne et al., 2002; Orbell \& Sheeran, 2000). In this exper- iment, to provide a strong instantiation of implementation intention encoding, we opted to use a combination of implementation intention instructions that previous laboratory studies had shown to be potent. In particular, we had the participants repeat the verbal implementation intention three times (following Cohen \& Gollwitzer, 2008), followed by $30 \mathrm{sec}$ of visual mental imagery of encountering the cue and performing the behavior (following Chasteen et al., 2001; McDaniel, Howard, \& Butler, 2008).

By contrast, as is usual, our typical prospective memory instruction did not explicitly force the participants to form a strong target-cue-intention linkage. The instructional wording avoided the "if-then" format (cf. Cohen $\&$ Gollwitzer, 2008), and the $30 \mathrm{sec}$ of visual imagery that followed (included to ensure that activating visual images per se was not driving implementation intention effects) required the participants to imagine performing the ongoing activity (not the prospective memory task). Accordingly, these typical prospective memory instructions provided less guidance in terms of intention encoding than did the implementation intention instructions. Consequently, at least some of the participants in this instructional condition might not spontaneously form a strong link between the target cue and the intention, a link that is explicitly emphasized in the implementation intention condition. Kliegel, Martin, McDaniel, Einstein, and Moor (2007) have provided evidence that participants' spontaneous prospective memory encodings when given typical prospective memory instructions do not necessarily focus sharply on the target-cue-intention link (see also Cohen \& Gollwitzer, 2008; McDaniel, Howard, \& Butler, 2008).

\section{Method}

Participants and Design. Sixty-four Washington University undergraduate students participated for course credit or monetary compensation. The participants were tested individually. Thirtytwo participants were randomly assigned to each of the prospective memory instruction groups (implementation intention or typical instructions); cognitive demand (standard or high) was varied within subjects.

Procedure. The participants first learned the instructions to the category decision task. They were told that they would have to decide whether a word in lowercase letters on the right side of the screen was a member of a (category) word in capital letters on the left side of the screen. The participants were further instructed to make their category decisions as quickly and accurately as possible by pressing the keys marked "Y" for yes or "N" for no ("5" and "6" on the number pad, respectively). Then participants were given 16 category decision practice trials, which were followed by the prospective memory task instructions. For the prospective memory task, participants were told that there was a secondary interest in their ability to remember to perform an action at a given point in the future and to press the " $Z$ " key if they ever saw either of two target words (corn and dancer; or in a counterbalance condition, fish and writer). The participants were told that their primary goal was still to respond to the category decision task.

After reading the prospective memory task instructions, the participants were asked to explain the instructions to the experimenter and then to imagine performing some aspect of their tasks for $30 \mathrm{sec}$. In the implementation intention condition, the participants were asked to say "When I see corn or dancer [or fish or writer] during the category decision task, I will press the ' $Z$ ' key" three times out loud. Then these participants were asked to imagine (for $30 \mathrm{sec}$ ) see- 
ing corn and dancer [ fish and writer] appear on the computer screen and pressing the " $Z$ " key. In the typical-instructions condition, the participants said 'I want to press ' $Z$ ' for corn and dancer [or fish and writer] during the category decision task" and then imagined performing the category decision task for $30 \mathrm{sec}$.

The participants filled out vocabulary and demographics information forms before continuing. They were then instructed that they would be performing the category decision task soon but that they would first be performing a lexical decision task (referred to as the speed task). The participants were instructed to decide whether a string of letters formed a word or a nonword as quickly and accurately as possible by pressing the keys marked "Y" or "N." The participants were further instructed that their only goal was to make word/nonword decisions and, if one of the target words appeared during the speed task, to only indicate that it was a word. The participants explained the lexical decision task instructions to the experimenter before performing a block of 20 lexical decision practice trials that included speed and accuracy feedback. Then the participants performed an experimental block of the lexical decision task that included 10 target items (e.g., corn and dancer), 10 control items (e.g., fish and writer), and 254 filler items ( 127 words and 127 nonwords). The filler items were the same as those used by Scullin, Einstein, and McDaniel (2009). Target and control items were separated by at least 3 filler items. We presented the target items during this phase for purposes not directly related to the present objectives ${ }^{1}$ (see Scullin et al., 2009, for a discussion relating to this particular aspect of the paradigm; see also Cohen, Dixon, \& Lindsay, 2005; Einstein et al., 2005, Experiment 5).

After performing the lexical decision task, the participants were instructed that they would be performing the category decision task again. They were further instructed that they would sometimes be asked to generate random numbers while performing the category decision task. The random-number generation task required saying a number (0-9) out loud to the beat of a metronome ( $1 \mathrm{click} / \mathrm{sec})$. The participants were given examples of the difference between "good" random sequences (e.g., 3, 6, 1, 0, 7, 4, 2, 8, 3, 5, 2) and "bad" random sequences (e.g., 1, 3, 5, 7, 6, 5, 4, 3, 3, 3, 3) and practiced generating numbers out loud for $30 \mathrm{sec}$. Then the participants performed four blocks of the category decision task, half under high cognitive demand (required to generate random numbers as well), and half under standard cognitive demand (did not generate random numbers). The order of these blocks was high, standard, high, standard (or the reverse, counterbalanced across participants). Each block included 24 category decision trials and 1 target trial. The target word appeared on Trials 22, 16, 24, and 21 in Blocks 1, 2, 3, and 4, respectively. The order of target presentation was corn ( fish), dancer (writer), corn (fish), dancer (writer), or the reverse (counterbalanced across participants). Each target item occurred on a yes trial (i.e., member of the same category) and a no trial (i.e., not a member of the same category). One target item was always a category label (dancer or writer), whereas the other target item was always a category member (corn or fish). Dancer and writer occurred with the (member) words ballerina and author. Corn and fish occurred with the (category) words vegetable and animal. Category decision filler items were the same as those used by McDaniel, Howard, and Butler (2008).

\section{Results}

For all the experiments, our statistical procedure for informing the a priori predictions developed in the introduction was to conduct a set of planned comparisons. This is a more direct approach to testing the present hypotheses than is computing omnibus ANOVAs (see e.g., Callender \& McDaniel, 2007, for a similar approach). To establish that the cognitive demand manipulation penalized performance, we contrasted the standard- and high-cognitivedemand conditions in the typical-instructions group.
Second, to evaluate the key question of whether implementation intentions buffer against decline in prospective memory under high cognitive demand, we compared the standard- and high-cognitive-demand conditions in the implementation intention group. Third, after conducting a comparison to confirm that the implementation intention effects emerged under standard-cognitive-demand conditions, we addressed whether implementation intention encoding improves prospective memory performance (relative to the typical encoding procedure) under high-cognitive-demand conditions. For consistency, this planned comparison approach was also adopted for all the ancillary measures reported. Significant effects were accompanied by estimates of effect size (Cohen's $d$; based on standard deviations of the means).

Prospective memory performance. We calculated the proportion of correct prospective memory responses in each experimental condition, and these means are displayed in Table 1. A prospective memory response was counted as correct if it occurred on the target trial or the following trial. Under the typical prospective memory encoding instructions, high cognitive demand produced significant declines in prospective remembering relative to standard cognitive demand $\left[F(1,62)=10.82, M S_{\mathrm{e}}=.10, p<.01\right.$, $d=0.64]$. Importantly, even with implementation intention encoding, prospective memory significantly declined under high-cognitive-demand conditions, as compared with standard-cognitive-demand conditions $[F(1,62)=$ $\left.22.88, M S_{\mathrm{e}}=.10, p<.001, d=0.89\right]$. As was expected, implementation intention encoding produced significantly better prospective remembering than did the typical encoding condition under standard cognitive demand $[F(1,62)=$ $\left.4.06, M S_{\mathrm{e}}=.10, p<.05, d=0.37\right]$. However, implementation intentions did not confer benefits (relative to the typical-instructions group) to prospective remembering in the high-cognitive-demand condition $(F<1)$.

Category decision task performance. We examined mean category decision reaction times on correct nontarget trials that were no greater than two standard deviations from each individual's mean (trimming was done separately for high- and standard-cognitive-demand blocks; Einstein et al., 2005). As is illustrated in Table 2, category decisions were slower during high-cognitive-demand blocks than during standard-demand blocks in both the

Table 1

Mean Proportions of Correct Prospective Memory Responses (With Standard Deviations) Across Conditions, Cognitive Demand, and Experiments

\begin{tabular}{|c|c|c|c|c|c|}
\hline \multirow[b]{3}{*}{ Experiment } & \multirow[b]{3}{*}{ Encoding Condition } & \multicolumn{4}{|c|}{ Cognitive Demand } \\
\hline & & \multicolumn{2}{|c|}{ Standard } & \multicolumn{2}{|c|}{ High } \\
\hline & & $M$ & $S D$ & $M$ & $S D$ \\
\hline \multirow[t]{2}{*}{1} & Implementation intention & .72 & .42 & .34 & .43 \\
\hline & Typical instructions & .56 & .45 & .30 & .36 \\
\hline \multirow[t]{3}{*}{2} & Implementation intention & .52 & .48 & .25 & .33 \\
\hline & Generate only & .63 & .42 & .33 & .41 \\
\hline & Typical instructions & .31 & .41 & .21 & .36 \\
\hline \multirow[t]{2}{*}{3} & Implementation intention & .58 & .46 & .17 & .35 \\
\hline & Practice & .54 & .49 & .35 & .45 \\
\hline
\end{tabular}


Table 2

Mean Trimmed Reaction Times (RTs, in Milliseconds) and Accuracy of Ongoing Task Performance (With Standard Deviations) Across Experiments As a Function of Cognitive Demand and Condition

\begin{tabular}{|c|c|c|c|c|c|c|c|c|}
\hline \multirow[b]{3}{*}{ Encoding Condition } & \multicolumn{4}{|c|}{$\begin{array}{c}\text { Standard Cognitive } \\
\text { Demand }\end{array}$} & \multicolumn{4}{|c|}{$\begin{array}{l}\text { High Cognitive } \\
\text { Demand }\end{array}$} \\
\hline & \multicolumn{2}{|c|}{ RTs } & \multicolumn{2}{|c|}{ Accuracy } & \multicolumn{2}{|c|}{ RTs } & \multicolumn{2}{|c|}{ Accuracy } \\
\hline & $M$ & $S D$ & $M$ & $S D$ & $M$ & $S D$ & $M$ & $S D$ \\
\hline \multicolumn{9}{|l|}{ Experiment 1} \\
\hline Implementation intention & 1,184 & 157 & .96 & .04 & 2,418 & 768 & .89 & .08 \\
\hline Typical instructions & 1,117 & 144 & .94 & .05 & 2,255 & 801 & .86 & .12 \\
\hline \multicolumn{9}{|l|}{ Experiment 2} \\
\hline Implementation intention & 1,075 & 145 & .95 & .04 & 2,519 & 774 & .90 & .07 \\
\hline Generate only & 1,175 & 182 & .94 & .04 & 2,484 & 738 & .90 & .05 \\
\hline Typical instructions & 1,049 & 134 & .95 & .03 & 2,475 & 851 & .91 & .04 \\
\hline \multicolumn{9}{|l|}{ Experiment 3} \\
\hline Implementation intention & 1,168 & 139 & .95 & .03 & 2,371 & 701 & .88 & .12 \\
\hline Practice & 1,280 & 214 & .95 & .05 & 2,601 & 943 & .86 & .12 \\
\hline
\end{tabular}

implementation intention and typical-instruction conditions $\left[F(1,62)=91.39, M S_{\mathrm{e}}=266,597.75, p<.001, d=\right.$ 2.23 , and $F(1,62)=77.72, M S_{\mathrm{e}}=266,597.75, p<.001$, $d=1.98$, respectively]. There were no significant differences between the prospective-memory instructional conditions in category decision responding during standardcognitive-demand $(F<1)$ and high-cognitive-demand $[F(1,62)=1.59, p=.21]$ blocks.

Performance on the category decision task was also assessed as the proportion of correct category decisions during standard-cognitive-demand and high-cognitivedemand blocks. The results paralleled the reaction time data and are displayed in Table 2. Category decision accuracy was worse during high-cognitive-demand blocks than standard-cognitive-demand blocks in the implementation intention condition $\left[F(1,62)=17.52, M S_{\mathrm{e}}=.005, p<\right.$ $.001, d=1.11]$ and the standard condition $[F(1,62)=$ $\left.25.35, M S_{\mathrm{e}}=.005, p<.001, d=0.87\right]$. Accuracy did not significantly differ between the instructional conditions during the standard-cognitive-demand $(F<1)$ and highcognitive-demand $[F(1,62)=3.01, p=.09]$ blocks.

Random-number generation performance. Following McDaniel, Howard, and Butler (2008), the ran- domness of number generation was assessed as redundancy (R), predictiveness of pair sequences (RNG score; Evans, 1978), and the equality or response alternative frequencies (turning point index [TPI]; Azouvi, Jokic, Van der Linden, Marlier, \& Bussel, 1996). There were no differences between encoding conditions for the $\mathrm{R}(F<1)$, RNG $(F<1)$, or TPI $[F(1,62)=2.20, p=.14]$ indices (see Table 3 ).

\section{Discussion}

Consistent with recent laboratory findings (Cohen \& Gollwitzer, 2008; McDaniel, Howard, \& Butler, 2008), when the demands of the ongoing activity were not overly challenging, implementation intention encoding significantly improved prospective remembering, relative to typical prospective memory instructions. Of central importance to the present objectives, under high cognitive demand, prospective memory declined substantially (about $50 \%$ or more) with both implementation intention and typical encoding instructions. Furthermore, under high cognitive demand, implementation intentions conferred no significant advantage to prospective memory, relative to typical encoding instructions. These patterns

Table 3

Mean Random-Number Generation Performance (With Standard Deviations) Across Condition and Evaluated by the R, RNG, and TPI Indexes

\begin{tabular}{|c|c|c|c|c|c|c|}
\hline \multirow[b]{2}{*}{ Encoding Condition } & \multicolumn{2}{|c|}{$\mathrm{R}$} & \multicolumn{2}{|c|}{ RNG } & \multicolumn{2}{|c|}{ TPI } \\
\hline & $M$ & $S D$ & $M$ & $S D$ & $M$ & $S D$ \\
\hline \multicolumn{7}{|l|}{ Experiment 1} \\
\hline Implementation intention & 3.19 & 2.44 & .35 & .06 & 86.29 & 9.46 \\
\hline Typical instructions & 3.62 & 2.81 & .35 & .07 & 82.47 & 11.11 \\
\hline \multicolumn{7}{|l|}{ Experiment 2} \\
\hline Implementation intention & 2.55 & 1.75 & .44 & .03 & 84.25 & 7.81 \\
\hline Generate only & 2.68 & 1.98 & .43 & .05 & 83.99 & 9.19 \\
\hline Typical instructions & 2.01 & 1.46 & .42 & .04 & 86.74 & 8.07 \\
\hline \multicolumn{7}{|l|}{ Experiment 3} \\
\hline Implementation intention & 3.52 & 2.95 & .35 & .06 & 85.68 & 10.35 \\
\hline Practice & 3.46 & 2.55 & .36 & .07 & 86.90 & 9.01 \\
\hline
\end{tabular}

Note - R, redundancy; RNG score, predictiveness of pair sequences; TPI, turning point index. 
disfavor the theoretical position that implementation intentions support automatized prospective memory responding (Cohen \& Gollwitzer, 2008; Gollwitzer, 1999).

These results are consistent with the idea that implementation intentions foster a strong associative encoding between the anticipated target cue and the intended action (McDaniel, Howard, \& Butler, 2008). Key to this interpretation is a critical distinction between the notion of spontaneous intention retrieval and the view of automatized prospective memory responding (as claimed for implementation intentions; Gollwitzer, 1999). Intention retrieval per se may not invariably lead to a prospective memory response. For instance, under conditions in which a number of responses are demanded (e.g., make a category decision under speeded conditions, as well as generate random number sequences), a participant may have to maintain the (spontaneously) retrieved intentionwhile scheduling and outputting other responses - before the intention can be executed (for further discussions, see Einstein et al., 1997; McDaniel et al., 1998). Prospective memory failures can occur because of difficulties in briefly maintaining the retrieved intention under high cognitive demands (Einstein, McDaniel, Williford, Pagan, \& Dismukes, 2003; see also McDaniel, Einstein, Graham, \& Rall, 2004). Consistent with this interpretation, implementation intentions, although favoring performance under standard-cognitive-demand conditions, did not advantage prospective memory in the present experiment under high-cognitive-demand conditions. Note that the automatized view of implementation intentions suggests that prospective memory responding is ballistically initiated when the environmental trigger is encountered and, thus, should not be penalized by high cognitive demand (Brandstätter et al., 2001; Gollwitzer, 1999).

\section{EXPERIMENT 2}

Because the extant literature has reported spared prospective memory performance under high cognitive demands after implementation intention encoding (McDaniel, Howard, \& Butler, 2008; see also Brandstätter et al., 2001), we conducted another experiment to replicate the Experiment 1 findings. A possible limitation to Experiment 1 was that the implementation intention involved linking the intended behavior to Cue Word A or Cue Word B. A reviewer raised the objection that the "or" phrase weakened the strength of the cue-intendedbehavior link. Accordingly, in this experiment, we limited the cue to one word, so that the implementation intention focused entirely on a single cue-word-intended-behavior link.

A second objective was to examine a generationencoding condition as an attempt to isolate the effects of strengthening the target-intended-action association on prospective memory (generating information in response to a cue strengthens the associative link between the cue and the generated information; Hirshman \& Bjork, 1988; McDaniel \& Waddill, 1990). Specifically, we required one group of participants to generate both components of the intention in the presence of the other component (generate the intended action given the target and generate the target given the intended action). In the implementation intention group, the participants not only generated components of the intention, but also encoded it, using an implementation intention (i.e., using an "if . . . then" framework). If implementation intentions confer advantages of automatized responding (Cohen \& Gollwitzer, 2008), advantages that theoretically go beyond good associative encoding of the target-intended-action (Bayer et al., 2009; Gollwitzer, 1999), the implementation intention group should display better prospective memory performance than the generate-only group. Furthermore, the implementation intention encoding should buffer against prospective memory decrements in the high-cognitive-demand condition, relative to the standard-cognitive-demand condition.

Alternatively, if implementation intention encoding primarily enhances associative encoding of the target and intended action, little should be gained in the presence of an encoding procedure that already stimulates such encoding (e.g., generation). From the perspective that implementation intention encoding primarily strengthens the target-intended-action association, the predictions are that (1) prospective memory will not differ significantly across the implementation intention and generate-only conditions; (2) both the implementation intention and generateonly conditions will improve prospective memory, relative to a typical-instructions condition under standard cognitive demands; and (3) as in Experiment 1, prospective memory will not be advantaged by implementation intentions (or by generation) under high-cognitive-demand conditions; instead, prospective memory performance will be disrupted (relative to standard cognitive demand).

\section{Method}

Participants and Design. Seventy-two Washington University undergraduates participated for monetary compensation or class credit. The participants were tested individually. Twenty-four participants were randomly assigned to each of the prospective memory instruction groups (typical instructions, generate only, or implementation intention); cognitive demand (standard or high) was varied within subjects.

Procedure. The procedure was similar to that in Experiment 1, in that a prospective memory task was embedded within a category decision task and attention was sometimes divided by having to perform a random-number generation task. Below, we highlight the features that differed from those in Experiment 1.

The participants first received the instructions to the randomnumber generation task, which was followed by a number generation practice phase. The instructions for this task were the same as those in Experiment 1. Next, the participants received the instructions for the category decision task, which they also practiced. The only difference between the category decision task in the present experiment and that in Experiment 1 was that the participants responded by pressing the "g" and " $h$ " keys (instead of " 5 " and " 6 " on the number pad). Then the participants were given the prospective memory task instructions to remember to press the Enter key if the word history appeared during the category decision task. The participants in the implementation intention condition were required to turn to the experimenter and say aloud "When I see the word history during the category decision task, I will press the Enter key." The participants in the typical-instructions and generate-only conditions simply read the prospective memory task instructions. Then the participants filled out demographics forms and took a vocabulary test. In the generate-only and implementation intention conditions, this delay 
was followed by a request to generate the prospective memory task instructions (in both the implementation intention and generate-only conditions) out loud in the following manner: "When I see the word history, I will ated the following answer: "When I ." The participants next generreread the prospective memory task instructions.

The participants next performed two experimental blocks of the category decision task that each consisted of 94 filler category decision trials and 2 target trials. During the high-cognitive-demand block (category decisions and random-number generation), the target occurred on Trials 16 and 57. During the standard-cognitivedemand block (only category decisions), the target appeared on Trials 38 and 60 .

\section{Results and Discussion}

Prospective memory performance. Correct prospective memory responses were tabulated in the same manner as in Experiment 1. As is shown in Table 1, and consistent with Experiment 1, prospective remembering in the implementation intention condition was significantly better than that in the typical-instructions condition under standard cognitive demand $\left[F(1,69)=6.26, M S_{\mathrm{e}}=.083, p=.01\right.$, $d=0.47]$, but not under high cognitive demand $(F<1)$. Likewise, prospective memory performance was better in the generate-only condition than in the typical-instructions condition under standard cognitive demand $[F(1,69)=$ $\left.12.33, M S_{\mathrm{e}}=.083, p<.001, d=0.77\right]$, but not under high cognitive demand $\left[F(1,69)=2.26, M S_{\mathrm{e}}=.083, p=\right.$ .14]. Importantly, the implementation intention condition did not significantly differ from (and was somewhat lower than) the generate-only condition under standard cognitive demand $\left[F(1,69)=1.56, M S_{\mathrm{e}}=.083, p=.22\right]$ and under high cognitive demand $(F<1)$. Therefore, even though an implementation intention encoding strategy produced better prospective remembering than did a typical encoding condition under standard cognitive demand, the benefits to prospective remembering did not extend beyond those obtained using a simple generation procedure.

Furthermore, the tests for automatic prospective memory responding in the implementation intention and generate-only conditions revealed that a high-cognitivedemand ongoing activity significantly attenuated prospective remembering (see Table 1). Prospective memory performance was lower during high-cognitive-demand blocks than during standard-cognitive-demand blocks in the implementation intention condition $[F(1,69)=10.62$, $\left.M S_{\mathrm{e}}=.083, p=.002, d=0.66\right]$ and in the generate-only condition $\left[F(1,69)=12.33, M S_{\mathrm{e}}=.083, p<.001, d=\right.$ $0.72]$. Prospective remembering also declined somewhat in the typical-instructions condition, but this decline did not reach statistical significance $\left[F(1,69)=1.59, M S_{\mathrm{e}}=\right.$ $.083, p=.21]$. Thus, implementation intention encoding of a single target-intended-action link, even when reinforced by requiring generation of components of the intention, did not produce automatized responding that protected prospective memory performance in the face of high attentional demands.

Category decision task performance. Both speed and accuracy of category decisions were evaluated in the same manner as in Experiment 1 (see Table 2).
There were no significant differences in mean trimmed reaction times between the conditions during standardcognitive-demand blocks or high-cognitive-demand blocks (all $F_{\mathrm{s}}<1$ ). However, responding was slower during high-cognitive-demand blocks, relative to standardcognitive-demand blocks, in the implementation intention condition $\left[F(1,69)=84.35, M S_{\mathrm{e}}=296,367.09, p<\right.$ $.001, d=2.59]$, the generate-only condition $[F(1,69)=$ $\left.69.36, M S_{\mathrm{e}}=296,367.09, p<.001, d=2.44\right]$, and the typical-instructions condition $\left[F(1,69)=82.37, M S_{\mathrm{e}}=\right.$ 296,367.09, $p<.001, d=2.34]$.

There were also no significant differences between conditions in the proportions of correct category decision trials during standard-cognitive-demand blocks ( $F \mathrm{~s}=2.35,1.20$, and $<1$ for the typical-instructions/ generate-only, implementation-intention/generate-only, and implementation-intention/typical-instructions comparisons, respectively) and high-cognitive-demand blocks ( $F \mathrm{~s}=2.03,1.20$, and $<1$ for the typical-instructions generate-only, implementation-intention/generate-only, and implementation-intention/typical-instructions comparisons, respectively; see Table 2). However, category decision accuracy was greater during standard-cognitivedemand than during high-cognitive-demand blocks in the implementation intention condition $[F(1,69)=23.23$, $\left.M S_{\mathrm{e}}=.001, p<.001, d=0.88\right]$, the generate-only condition $\left[F(1,69)=16.43, M S_{\mathrm{e}}=.001, p<.001, d=0.88\right]$, and the typical-instructions condition $[F(1,69)=17.33$, $\left.M S_{\mathrm{e}}=.001, p<.001, d=1.13\right]$.

Random-number generation performance. There were no significant differences between conditions in random-number generation performance, measured as $\mathrm{R}\left(F_{\mathrm{S}}=1.80,1.17\right.$, and $<1$, for the typical-instructions/ generate-only, implementation-intention/typicalinstructions, and implementation-intention/generate-only comparisons, respectively), $\mathrm{RNG}\left(F_{\mathrm{S}}=1.18,2.4\right.$, and $<1$ for the typical-instructions/generate-only, implementationintention/typical-instructions, and implementationintention/generate-only comparisons, respectively), and TPI $(F \mathrm{~s}=1.29,1.05$, and $<1$ for the typical-instructions/ generate-only, implementation-intention/typicalinstructions, and implementation-intention/generate-only comparisons, respectively).

In sum, the implementation intention (plus generation) condition produced performance profiles that replicated those in Experiment 1 and, furthermore, were quite similar to those in the generate-only condition. In particular, both conditions significantly improved prospective memory, relative to a typical-instructions condition, under standard cognitive demands. However, in the implementation intention and generate-only conditions, prospective memory responding was significantly impaired under high cognitive demands, relative to the standard cognitive demands. Furthermore, these conditions produced no performance gains, relative to the typical-instructions condition, under high cognitive demands. These results, along with those from Experiment 1, are not consistent with the theoretical assertion that implementation intentions produce automatic prospective memory responding (Gollwitzer, 1999). In this regard, it is noteworthy that in 
this experiment, the particular implementation intention encoding could not be considered a "weak" instantiation of an implementation intention, because a single target cue was used and generation was incorporated into the implementation intention.

The findings are, however, consistent with the interpretation that forming an implementation intention strengthened the target-intended-action association. First, an implementation intention did not confer any advantage to prospective memory beyond that produced by an encoding condition specifically oriented to strengthening the target-intended-action association (generation encoding of the prospective memory cue and intention). Second, as was discussed earlier, a stronger target-intended-action association would be expected to enhance prospective memory (relative to a typical-instructions encoding), but not necessarily under high cognitive demand. This expectation was confirmed by the pattern of prospective memory effects in the generate-only condition, effects that were identical to those produced by the implementation intention condition.

\section{EXPERIMENT 3}

Our final experiment was an initial attempt to evaluate a related premise of the claim that implementation intentions support automatic action initiation. The premise is that "implementation intentions create instant habits" (Gollwitzer, 1999, p. 499). ${ }^{2}$ Here, rather than evaluate habit formation per se, we focused on whether an implementation intention creates a representation that is functionally similar to that accrued from behavioral practice. We directly compared a practice group with the implementation intention group (having established the effectiveness of our implementation intention encoding under the standard-cognitive-demand condition in the two previous experiments, we opted not to repeat the typicalinstructions condition). In the practice group, the participants were given a block of ongoing task practice trials in which the prospective memory target cue appeared several times, and the participants were instructed to try to form a habit of performing the associated (prospective memory) response each time they saw the target. As far as we know, this is the first experimental study of how implementation intention encoding fares relative to (experimentally manipulated) behavioral practice in supporting prospective memory performance (but see Aarts \& Dijksterhuis, 2000 , for a similar attempt using an individual-differences approach). If practice leads to significantly higher levels of prospective memory performance than does implementation intention encoding in the high-cognitive-demand condition, it would further disfavor the claim that implementation intention encoding "is as effective in automatizing action initiation as repeated and consistent practice" (Gollwitzer, 1999, p. 499).

\section{Method}

Participants and Design. Forty-eight Washington University undergraduate students were tested individually and received monetary compensation or class credit for their participation. Twenty-four participants were randomly assigned to each of the implementation intention and practice groups; cognitive demand (standard or high) was varied within subjects.

Procedure. The procedure was identical to that in Experiment 1, with few exceptions. The participants learned about the category decision and prospective memory tasks first. The present experiment diverged from Experiment 1 in that a 30-trial category decision practice block followed the prospective-memory-encoding (instruction) phase. In the practice condition (which was identical to Experiment 1's typical-instructions condition up to this point), the participants were told that their target words would appear during the practice block and that they should try to form a habit of pressing the "Z" key when their target words appeared. They were further instructed that if a habit was formed, they should not have to keep their target words in mind; instead, seeing the target word should reflexively remind them to press the "Z" key. During this additional practice block, each target word appeared five times (total of 10 target practice trials). During the practice block, half of the target words appeared as category labels, and half appeared as category members: The target word dancer (or writer) appeared with the (member) word mirror, and the target word corn (or fish) appeared with the (category) term food type. In the implementation-intention condition, the participants were told only that they would be practicing the category decision task again; for these participants, the prospective memory target words did not appear during the practice block.

Following the practice block, the participants filled out vocabulary and demographics forms (identical to those in Experiment 1) before performing the lexical decision and category decision tasks. These tasks were identical to those in Experiment 1.

\section{Results and Discussion}

Prospective memory performance. Replicating the previous experiments, the implementation intention condition showed lower prospective memory performance under high cognitive demand than under standard cognitive demand $\left[F(1,46)=25.53, M S_{\mathrm{e}}=.08, p<.001, d=\right.$ 1.00]. Similarly, in the practice group, performance was significantly lower during high-cognitive-demand blocks than during standard-cognitive-demand blocks $[F(1,46)=$ $\left.5.48, M S_{\mathrm{e}}=.08, p=.02, d=0.40\right]$. Importantly, whereas there was no prospective memory difference between conditions during the standard-cognitive-demand blocks $(F<1)$, during high-cognitive-demand blocks, prospective memory performance was significantly greater in the practice condition than in the implementation intention condition $\left[F(1,46)=4.92, M S_{\mathrm{e}}=.08, p=.03, d=0.45\right.$; see Table 1]. Thus, practicing the prospective memory task was superior to forming an implementation intention in supporting prospective memory performance under high cognitive demands.

Category decision task performance. Category decisions were slower during high-cognitive-demand blocks than during standard-cognitive-demand blocks in both the implementation intention condition $[F(1,46)=48.57$, $\left.M S_{\mathrm{e}}=357,557.54, p<.001, d=2.38\right]$ and the practice condition $\left[F(1,46)=58.57, M S_{\mathrm{e}}=357,557.54, p<\right.$ $.001, d=1.93]$. Speed of category decisions was similar between conditions during blocks with standard cognitive demand $(F<1)$ and blocks with high cognitive demand $\left[F(1,46)=1.78, M S_{\mathrm{e}}=357,557.54, p=.19\right]$.

Category decision task accuracy was evaluated as the proportion of correct trials. Consistent with the reaction time data, category decision accuracy was lower during 
high-cognitive-demand blocks than during standardcognitive-demand blocks in the implementation intention condition $\left[F(1,46)=8.40, M S_{\mathrm{e}}=.007, p=.006, d=\right.$ $0.80]$ and the practice condition $\left[F(1,46)=13.89, M S_{\mathrm{e}}=\right.$ $.007, p<.001, d=0.98]$. Furthermore, there were no accuracy differences between conditions during either highcognitive-demand or standard-cognitive-demand blocks (both $F_{\mathrm{s}}<1$ ).

Random-number generation performance. There were no significant differences between conditions when examining random number generation performance using R, RNG, or TPI (all $F \mathrm{~s}<1$ ).

For every measure, ongoing task performances were comparable for the practice and the implementation intention conditions. Thus, the superior prospective memory performance in the practice group, relative to the implementation intention group (in the high-cognitive-demand condition), was not due to participants in the practice condition sacrificing ongoing task performance to support better prospective memory performance.

In sum, the prospective memory results provide direct evidence that implementation intention encoding does not provide the same kinds of benefits as repeated practice (cf. Gollwitzer, 1999). Indeed, this was the case even though the present practice regimen did not completely protect prospective memory against the increased cognitive demand (i.e., from generating random numbers). Thus, it is unlikely that the present practice regimen was sufficient to produce a habitlike response, a finding that underscores the improbability of producing habits with a simple "if . . . then" encoding strategy (see Gollwitzer, 1999). The critical point is that the present practice regimen, though not necessarily leading to habitlike responding, was sufficient to produce prospective memory performance superior to that observed for implementation intentions under high cognitive demand. Accordingly, implementation intention encoding, at least as instantiated in the extant literature, does not appear to achieve the same prospective memory dynamics as does practice, much less a habit (cf. Gollwitzer, 1999). ${ }^{3}$

\section{GENERAL DISCUSSION}

Implementation intention encoding has been shown to be extremely effective in improving the likelihood that people will perform intended actions at appropriate moments in the future, such as remembering to take a daily vitamin C tablet over the course of several weeks (Sheeran \& Orbell, 1999), to initiate breast self-examinations (Orbell, Hodgkins, \& Sheeran, 1997), and to monitor blood glucose levels (Liu \& Park, 2004; see Gollwitzer, 1999, for a more extensive review). At issue in the present study was the theoretical claim that the benefits of implementation intentions rest, at least in part, on their ability to produce automaticity in prospective memory responding (Gollwitzer, 1999; see also Liu \& Park, 2004).

As has been discussed throughout this article, the present results consistently disfavor this theoretical claim that implementation intention encoding automatizes prospective memory responding (Gollwitzer, 1999, p. 499).
Countering the notion of automaticity, implementation intention encoding failed across three experiments to buffer prospective memory against the negative effects of high cognitive demand. Importantly, this failure (to buffer prospective memory against the effects of high cognitive demands) cannot be attributed to a substandard or weak implementation intention encoding. Experimental instantiations of implementation intention encoding have varied from verbal repetition of the "if . . then" statement (Bayer et al., 2009, Study 2; Cohen \& Gollwitzer, 2008) to an embellished (full) procedure that includes the "if . . . then" statement plus visual imagery directed at reinforcing the target-cue-intended-behavior link (Chasteen et al., 2001; Liu \& Park, 2004). In the present Experiment 1, the implementation intention encoding required both the verbal statement and visual imagery, and in Experiment 2, the implementation intention encoding was augmented by generation. Both of these instantiations of implementation intention encoding produced the expected prospective memory advantage, relative to a typical prospective memory instruction condition.

Past reports that showed that completion of intentions did not suffer under high cognitive demand with implementation intention encoding may not have created sufficiently high cognitive demand. As was noted in the introduction of this article, in the studies by Brandstätter et al. (2001) and McDaniel, Howard, and Butler (2008, Experiment 1 ), the cognitive demand manipulations may not have been entirely successful, since prospective memory (assessed by speed of performance in Brandstätter et al., 2001) sometimes did not decline in the typical-instructions condition (a familiarization control in Brandstätter et al., 2001 ) in the high-cognitive-demand condition. Even in McDaniel, Howard, and Butler's Experiment 2, wherein the high-cognitive-demand condition involved generating random digits, the prospective memory performances were quite high (ranging from $82 \%$ to $95 \%$ ), possibly suggesting that the experimenter's pacing of the primary ongoing activity (words presented at a 5-sec rate), the subjective nature of the task (rating word qualities), or both, allowed the participants in the high-cognitive-demand condition to allocate resources to the prospective memory task (discussed in more detail below). In contrast, in the present paradigm, the primary ongoing task was speeded and required more objective responses, thereby making it more difficult for the participants under high cognitive demand to marshal resources to assist with the prospective memory task. Thus, the cognitive demand appears to be more challenging in this study than in McDaniel, Howard, and Butler and, thus, provided a more telling test of the degree to which implementation intention encoding produces automatized responding.

Another telling finding was that in the high-cognitivedemand conditions, prospective memory responding under implementation intentions did not reach the levels produced by practice (Experiment 3). To our knowledge, this is the first experimental study to evaluate the parallels between prospective memory performance after implementation intention encoding and after practice (cf. Aarts $\&$ Dijksterhuis, 2000). The advantage of the practice group 
was not a consequence of a resource allocation policy that favored the prospective memory task over the ongoing tasks. Such a resource allocation policy would have been expected to penalize ongoing task performances (cf. McDaniel, Einstein, \& Rendell, 2008). Yet, ongoing task performance of the practice group did not differ statistically from that of the implementation intention group. These patterns, taken in concert, suggest that implementation intention encoding, as least as currently formulated in the literature, does not necessarily create functional equivalence with behavioral practice (Gollwitzer, 1999), and does not confer automatic action control that is akin to "instant habits" (Cohen \& Gollwitzer, 2008, p. 384).

One alternative theoretical interpretation of the present patterns is that implementation intention encoding imbues the prospective memory task with more importance than the typical prospective memory instructions (i.e., Experiments 1 and 2), but the greater perceived importance does not buffer against high cognitive demands. The idea here is that as the prospective memory task increases in importance, more available resources will be devoted to the prospective memory task, with concomitant improvements in performance (e.g., Kliegel, Martin, McDaniel, \& Einstein, 2004). On this interpretation, ongoing task performances should also decline (for the implementation intention condition), since resources will be reallocated from the ongoing tasks to the prospective memory task (see Kliegel, Martin, McDaniel, \& Einstein, 2001, 2004). This pattern did not emerge, however.

The present results seem most consistent with the view that implementation intention encoding (as operationalized in the present study with a direct "if . . . then" statement linking the cue and the intention and reinforced with visual imagery or generation in several experiments) likely enhances the associative encoding between an anticipated environmental cue and an intended action. ${ }^{4}$ Indeed, given the range of encoding procedures in the literature that have been used to instantiate implementation intentions (cf. Brandstätter et al., 2001; Chasteen et al., 2001; Gollwitzer \& Brandstätter, 1997; Milne et al., 2002), the term seems to have broadened beyond the explicit idea of an "if . . . then" format (Cohen \& Gollwitzer, 2008) to a range of encoding procedures that could strengthen the associative link between the target cue and the intended behavior. An enhanced associative encoding would be expected to increase prospective memory performance according to views that suggest that prospective memory is, in part, supported by a spontaneous retrieval process that delivers an intention to awareness when a target environmental cue is encountered (see McDaniel \& Einstein, 2007; McDaniel, Guynn, et al., 2004; it may be possible that an enhanced associative encoding could also increase the likelihood that the target cue could be recognized as such-e.g., Smith, 2003).

Importantly, however, a key aspect of the spontaneous retrieval view, and one that diverges from the Gollwitzer (1999; see also Cohen \& Gollwitzer, 2008) formulation, is that spontaneous retrieval does not necessarily imply a completely automatized prospective memory response (Einstein \& McDaniel, in press; Einstein et al., 1997; note that this is a modification of our original supposition that spontaneous retrieval could be equated with automatic prospective memory performance; McDaniel, Guynn, et al., 2004). This view accommodates our central finding that implementation intention encoding did not buffer prospective memory against very highly demanding ongoing activities. For example, secondary tasks (in a high-cognitive-demand situation) may interfere with full processing of the cue, which theoretically would disrupt spontaneous associative retrieval (Moscovitch, 1994). Varying thresholds for allowing cue-driven thoughts (e.g., prospective memory intentions) into consciousness (cf. Norman, Newman, \& Detre, 2007) could also influence the success of spontaneous retrieval of the intended action. Specifically, as secondary tasks are added to ongoing activities, thereby requiring more attentional resources, individuals may set a higher threshold for allowing cue-driven thoughts unrelated to the ongoing tasks to enter consciousness. This notion is consistent with the idea that spontaneously retrieved memories (memories that occur without a deliberate search) are more likely to reach consciousness when we are relaxed and our attention is not highly focused (Ball \& Little, 2006; Kvavilashvili \& Mandler, 2004). Even if retrieval were not challenged by demanding ongoing activities, demanding ongoing activities could compromise execution of the prospective memory intention by interfering with its maintenance in awareness until execution was possible (see Einstein et al., 2003).

In sum, implementation intention encoding did not maintain prospective remembering under the challenging ongoing task situations reflected in the present study and did not mimic the effects of behavioral practice in supporting prospective memory under these challenging ongoing task situations (shown in Experiment 3). Therefore, implementation intention encoding, at least as instantiated in this and other studies, cannot be viewed as creating an automatic prospective memory response.

\section{AUTHOR NOTE}

We thank Daniel Howard for conducting Experiment 2. We also thank Jake Fantus, Matthew Kowalski, Laura Cobb, Marco Chacon, and Michael Clerkin for their help with data collection and analysis. We appreciate helpful comments from Richard Marsh on an earlier version of the manuscript. Correspondence concerning this article should be addressed to M. A. McDaniel, Department of Psychology, Washington University, One Brookings Drive, St. Louis, MO 63130-4899 (e-mail: mmcdanie@, artsci.wustl.edu).

\section{REFERENCES}

Aarts, H., \& Dijksterhuis, A. (2000). Habits as knowledge structures: Automaticity in goal-directed behavior. Journal of Personality \& Social Psychology, 78, 53-63.

Azouvi, P., Jokic, C., Van der Linden, M., Marlier, N., \& Bussel, B. (1996). Working memory and supervisory control after severe closedhead injury: A study of dual task performance and random generation. Journal of Clinical \& Experimental Neuropsychology, 18, 317-337.

Ball, C. T., \& Little, J. C. (2006). A comparison of involuntary autobiographical memory retrievals. Applied Cognitive Psychology, 20, 1167-1179.

Bayer, U. C., Achtziger, A., Gollwitzer, P. M., \& Moskowitz, G. B. (2009). Responding to subliminal cues: Do if-then plans facili- 
tate action preparation and initiation without conscious intent? Social Cognition, 27, 183-201.

Brandstätter, V., Lengfelder, A., \& Gollwitzer, P. M. (2001). Implementation intentions and efficient action initiation. Journal of Personality \& Social Psychology, 81, 946-960.

Callender, A. A., \& McDaniel, M. A. (2007). The benefits of embedded question adjuncts for low and high structure builders. Journal of Educational Psychology, 99, 339-348.

Chasteen, A. L., Park, D. C., \& Schwarz, N. (2001). Implementation intentions and facilitation of prospective memory. Psychological Science, 12, 457-461.

Cohen, A.-L., Dixon, R. A., \& Lindsay, D. S. (2005). The intention interference effect and aging: Similar magnitude of effects for young and old adults. Applied Cognitive Psychology, 19, 1177-1197.

Cohen, A.-L., \& Gollwitzer, P. M. (2008). The cost of remembering to remember: Cognitive load and implementation intentions influence ongoing task performance. In M. Kliegel, M. A. McDaniel, \& G. O. Einstein (Eds.), Prospective memory: Cognitive, neuroscience, developmental, and applied perspectives (pp. 367-390). Mahwah, NJ: Erlbaum.

Einstein, G. O., \& McDaniel, M. A. (in press). Prospective memory and what costs do not reveal about retrieval processes: A commentary on Smith, Hunt, McVay, and McConnell (2007). Journal of Experimental Psychology: Learning, Memory, \& Cognition.

Einstein, G. O., McDaniel, M. A., Thomas, R., Mayfield, S., Shank, H., Morrisette, N., \& Breneiser, J. (2005). Multiple processes in prospective memory retrieval: Factors determining monitoring versus spontaneous retrieval. Journal of Experimental Psychology: General, 134, 327-342.

Einstein, G. O., McDaniel, M. A., Williford, C. L., Pagan, J. L., \& Dismukes, R. K. (2003). Forgetting of intentions in demanding situations is rapid. Journal of Experimental Psychology: Applied, 9, 147-162.

Einstein, G. O., Smith, R. E., McDaniel, M. A., \& Shaw, P. (1997) Aging and prospective memory: The influence of increased task demands at encoding and retrieval. Psychology \& Aging, 12, 479-488.

Evans, F. J. (1978). Monitoring attention deployment by random number generation: An index to measure subjective randomness. Bulletin of the Psychonomic Society, 12, 35-38.

GolLWITZER, P. M. (1999). Implementation intentions: Strong effects of simple plans. American Psychologist, 54, 493-503.

Gollwitzer, P. M., \& Brandstätter, V. (1997). Implementation intentions and effective goal pursuit. Journal of Personality \& Social Psychology, 73, 186-199.

Hirshman, E., \& BJork, R. A. (1988). The generation effect: Support for a two-factor theory. Journal of Experimental Psychology: Learning, Memory, \& Cognition, 14, 484-494.

Kliegel, M., Martin, M., McDaniel, M. A., \& Einstein, G. O. (2001). Varying the importance of a prospective memory task: Differential effects across time- and event-based prospective memory. Memory, 9, 1-11.

Kliegel, M., Martin, M., McDaniel, M. A., \& Einstein, G. O. (2004). Importance effects on performance in event-based prospective memory tasks. Memory, 12, 553-561.

Kliegel, M., Martin, M., McDaniel, M. A., Einstein, G. O., \& Moor, C. (2007). Realizing complex delayed intentions in young and old adults: The role of planning aids. Memory \& Cognition, 35, 17351746.

Kvavilashvili, L., \& Mandler, G. (2004). Out of one's mind: A study of involuntary semantic memories. Cognitive Psychology, 48, 47-94.

LiU, L. L., \& PARK, D. C. (2004). Aging and medical adherence: The use of automatic processes to achieve effortful things. Psychology \& Aging, 19, 318-325.

Marsh, R. L., \& Hicks, J. L. (1998). Event-based prospective memory and executive control of working memory. Journal of Experimental Psychology: Learning, Memory, \& Cognition, 24, 336-349.

Marsh, R. L., Hicks, J. L., \& WATson, V. (2002). The dynamics of intention retrieval and coordination of action in event-based prospective memory. Journal of Experimental Psychology: Learning, Memory, \& Cognition, 28, 652-659.

McDaniel, M. A., \& Einstein, G. O. (2007). Prospective memory: An overview and synthesis of an emerging field. Thousand Oaks, CA: Sage.
McDaniel, M. A., Einstein, G. O., Graham, T., \& Rall, E. (2004) Delaying execution of intentions: Overcoming the costs of interruptions. Applied Cognitive Psychology, 18, 533-547.

McDaniel, M. A., Einstein, G. O., \& Rendell, P. G. (2008). The puzzle of inconsistent age-related declines in prospective memory: A multiprocess explanation. In M. Kliegel, M. A. McDaniel, \& G. O. Einstein (Eds.), Prospective memory: Cognitive, neuroscience, developmental, and applied perspectives (pp. 141-160). Mahwah, NJ: Erlbaum.

McDaniel, M. A., Guynn, M. J., Einstein, G. O., \& Breneiser, J. (2004). Cue-focused and reflexive-associative processes in prospective memory retrieval. Journal of Experimental Psychology: Learning, Memory, \& Cognition, 30, 605-614.

McDaniel, M. A., Howard, D. C., \& Butler, K. M. (2008). Implementation intentions facilitate prospective memory under high attention demands. Memory \& Cognition, 36, 716-724.

McDaniel, M. A., Robinson-Riegler, B., \& Einstein, G. O. (1998). Prospective remembering: Perceptually driven or conceptually driven processes? Memory \& Cognition, 26, 121-134.

McDaniel, M. A., \& WAdDILl, P. J. (1990). Generation effects for context words: Implications for item-specific and multifactor theories. Journal of Memory \& Language, 29, 201-211.

Milne, S., Orbell, S., \& Sheeran, P. (2002). Combining motivational and volitional interventions to promote exercise participation: Protection motivation theory and implementation intentions. British Journal of Health Psychology, 7, 163-184.

Moscovitch, M. (1994). Memory and working with memory: Evaluation of a component process model and comparisons with other models. In D. L. Schacter \& E. Tulving (Eds.), Memory systems (pp. 269310). Cambridge, MA: MIT Press.

Norman, K. A., Newman, E. L., \& Detre, G. (2007). A neural network model of retrieval-induced forgetting. Psychological Review, 114, 887-953.

Orbell, S., Hodgkins, S., \& Sheeran, P. (1997). Implementation intentions and the theory of planned behavior. Personality \& Social Psychology Bulletin, 23, 945-954.

Orbell, S., \& Sheeran, P. (2000). Motivational and volitional processes in action initiation: A field study of the role of implementation intentions. Journal of Applied Social Psychology, 30, 780-797.

Scullin, M. K., Einstein, G. O., \& McDaniel, M. A. (2009). Evidence for spontaneous retrieval of suspended but not finished prospective memories. Memory \& Cognition, 37, 425-433.

SheEran, P., \& Orbell, S. (1999). Augmenting the theory of planned behavior: Roles for anticipated regret and descriptive norms. Journal of Applied Social Psychology, 29, 2107-2142.

SмiтH, R. E. (2003). The cost of remembering in event-based prospective memory: Investigating the capacity demands of delayed intention performance. Journal of Experimental Psychology: Learning, Memory, \& Cognition, 29, 347-361.

WinOGRAD, E. (1988). Some observations on prospective remembering. In M. M. Gruneberg, P. E. Morris, \& R. N. Sykes (Eds.), Practical aspects of memory: Current research and issues (Vol. 1, pp. 348-353). Chichester, U.K.: Wiley.

\section{NOTES}

1. Although the focus of the present research was to examine prospective memory performance, we also investigated whether the presentation of the prospective memory cue during an ongoing task in which the prospective task was not to be performed (lexical decision) would slow responding for that ongoing task (termed the intention interference effect; Cohen et al., 2005; Einstein et al., 2005; Scullin et al., 2009). For the sake of efficiency, we examined the intention interference results collapsed for Experiments 1 and 3 (the Experiment 2 method did not allow for this analysis). Consistent with previous research (Einstein et al., 2005; Scullin et al., 2009), we trimmed reaction times for each participant to include only correct lexical decision responses that were fewer than two standard deviations away from the individual's mean.

In the typical-instructions condition (Experiment $1 ; n=32$ ), responding was significantly slower on target trials $(M=489 \mathrm{msec})$ than on control trials $(M=471 \mathrm{msec})\left[F(1,109)=4.61, M S_{\mathrm{e}}=1,125.30, p=.03\right]$, thereby indicating that intention interference obtained in the standard condition. The intention interference effect also obtained in the implementa- 
tion intention conditions (Experiments 1 and $3 ; n=56$ ). Responding was significantly slower on target trials $(M=502 \mathrm{msec})$ than on control trials $(M=482 \mathrm{msec})\left[F(1,109)=9.95, M S_{\mathrm{e}}=1,125.30, p<.01\right]$. By contrast, in the practice condition, the intention interference effect was eliminated (Experiment $3 ; n=24$ ), since there were similar response latencies to the target $(M=522 \mathrm{msec})$ and control $(M=520 \mathrm{msec})$ trials $(F<1)$. The intention interference effect results in the typical-instructions and implementation intention conditions replicated the findings of previous research (Einstein et al., 2005; Scullin et al., 2009).

2. Gollwitzer (1999; see also Cohen \& Gollwitzer, 2008) does not provide a clear theoretical explanation for how implementation intention encoding could produce a response similar in nature to that produced by a habit; however, the implication seems to be that an implementation intention promotes a (sufficiently) strong mental link between an environmental cue and the intended action that can substitute for (or mimic) the presumed linkages between cue and action that are forged through repeated practice.

3. One reviewer objected that implementation intentions and practice were not put on equal footing in this experiment, because the implementation intention encoding was not repeated numerous times, whereas, by definition, practice was repeated. This objection, however, does no capture the essence of the extant theoretical claims and empirical literature. The theoretical conjecture has been that an implementation intention produces an "instant habit, as it originates from a single [italics added] act of will rather than being produced by repeated and consistent selection of a certain course of action in the same situation" (Cohen \& Gollwitzer, 2008, p. 384). Moreover, the empirical findings regarding implementation intentions on which this theoretical framework has appealed for support have depended on few, if any, repetitions of the implementation intention encoding (Brandstätter et al., 2001; Chasteen et al., 2001; McDaniel, Howard, \& Butler, 2008; Orbell \& Sheeran, 2000; Sheeran \& Orbell, 1999). As far as we are aware, implementation intention encoding procedures have, at most, required three repetitions of the implementation intention statement (e.g., Cohen \& Gollwitzer, 2008) or a relatively short period of mentally applying the once-presented implementation intention (e.g., Chasteen et al., 2001; see also Liu \& Park, 2004). These procedures have been assumed to produce strong mental if-then links (at least implicitly, since this literature has helped fuel and support the theoretical claims regarding habitlike automaticity associated with implementation intentions; see, e.g., Cohen \& Gollwitzer, 2008; Gollwitzer, 1999). The implementation intention encoding in this experiment was based on some of the strongest procedures evidenced in the literature - that is, three verbal repetitions and $30 \mathrm{sec}$ of mentally applying the intention. It might be the case that many more repetitions of the implementation intention encoding could support prospective memory performance that mimics the effects of extensive practice, but current conceptualizations of implementation intentions do not specify that frequent repeated encodings should be an essential feature of implementation intention effects.

4. Specification of a particular environmental cue, formulation of a particular intended behavior (rather than a general goal), or both could produce the benefits, at least in part, found for implementation intentions in the everyday situations examined in much of the research with implementation intentions (see Gollwitzer, 1999). In laboratory prospective memory tasks, however, these components are already present in the typical prospective memory instructions. Moreover, in many laboratory prospective memory tasks, the ongoing activity in which the prospective memory task is embedded stimulates full processing of the prospective memory cue (in which case, the cue is often termed a focal cue; Einstein et al., 2005), thereby theoretically enhancing detection of that target cue. Consequently, the use of focal prospective memory cues in laboratory studies, as in the present study, would presumably facilitate detection of the target cue in the environment, a mechanism that might also underlie the benefits of implementation intentions in everyday situations (but not necessarily those observed in the present laboratory study).

(Manuscript received April 14, 2009; revision accepted for publication September 13, 2009.) 